\title{
MÉTODO SIMPLES PARA QUANTIFICAR O METABOLISMO AERÓBIO E ANAERÓBIO DE LEVEDURA ALCOÓLICA
}

\author{
WALDEMAR GASTONI VENTURINI FILHO* \\ LUCIANA TREVISAN BRUNELLI** \\ JULIANO TONIATO*** \\ TOSHIO NOJIMOTO**** \\ FERNANDO VALADARES NOVAES ${ }^{* * * * *}$
}

\begin{abstract}
O objetivo deste trabalho foi quantificar o metabolismo aeróbio (respiração) e anaeróbio (fermentação alcoólica) de levedura, durante a fermentação de mostos de melaço e de caldo de cana por meio de método estequiométrico. $O$ experimento foi conduzido com três tratamentos (teor de sólidos solúveis dos mostos) e três repetições, perfazendo nove parcelas experimentais para cada tipo de matéria-prima (melaço ou caldo de cana). Diluiu-se o melaço com água para produzir mostos com 10, 20 e $30^{\circ}$ Brix, enquanto que o teor de sólidos solúveis do caldo foi corrigido com água ou açúcar cristal com o mesmo propósito. Os mostos foram inoculados com levedura seca de panificação (100 g/L) e fermentados à temperatura ambiente. Foram determinadas as massas de $\mathrm{CO}_{2}$ e de etanol produzidos durante o processo fermentativo para quantificar estequiometricamente o açúcar metabolizado (fermentado e respirado) pela levedura. Os resultados mostraram que a levedura catabolizou o açúcar, durante o processo fermentativo, tanto por via aeróbia como pela anaeróbia com prevalência dessa última. A taxa de fermentação no mosto de melaço ficou entre $80,02 \%$ (10 ${ }^{\circ}$ Brix) e $91,58 \%$ (30 ${ }^{\circ}$ Brix), enquanto no mosto de caldo de cana esses valores foram de $83,17 \%$ (10 ${ }^{\circ}$ Brix) e 89,16\% (30 ${ }^{\circ}$ Brix) indicando a presença do efeito Crabtree.
\end{abstract}

PALAVRAS-CHAVE: FERMENTAÇÃO; LEVEDURA; EFEITO PASTEUR; EFEITO CRABTREE.

* Doutor em Agronomia, Professor, Faculdade de Ciência Agronômicas, Universidade Estadual Paulista (UNESP), Botucatu, SP (e-mail: venturini@fca.unesp.br).

** Mestre em Agronomia, Doutoranda, Faculdade de Ciências Agronômicas, UNESP, Botucatu, SP (e-mail: Itbrunelli@fca.unesp.br).

*** Biólogo, Mestrando em Energia na Agricultura, Faculdade de Ciências Agronômicas, UNESP, Botucatu, SP (e-mail: jtoniato@fca.unesp.br).

**** Doutor em Agronomia, Professor, Faculdade de Ciências Agronômicas, UNESP, Botucatu, SP (e-mail: toshio@fca.unesp.br).

***** Doutor em Agronomia, Professor, Escola Superior de Agricultura Luiz de Queiroz, Universidade de são Paulo, Piracicaba, SP (e-mail: fvnovaes@terra.com.br). 


\section{INTRODUÇÃo}

As leveduras alcoólicas da espécie Saccharomyces cerevisiae utilizam açúcares como substrato para seu metabolismo. Classificadas como micro-organismos facultativos, podem catabolizar açúcares para a produção de energia química celular (ATP) pelas vias aeróbia e anaeróbia. No primeiro caso, conhecido como respiração, uma molécula de sacarose é oxidada na presença de oxigênio, resultando em gás carbônico e água nas quantidades estequiométricas mostradas na equação 1 (LEHNINGER, 2002):

\begin{tabular}{|c|c|c|c|c|c|}
\hline $\begin{array}{c}\mathrm{C}_{12} \mathrm{H}_{22} \mathrm{O}_{11} \\
342\end{array}$ & $\begin{array}{c}\mathrm{H}_{2} \mathrm{O} \\
18\end{array}$ & $\rightarrow$ & $\begin{array}{c}\mathrm{C}_{6} \mathrm{H}_{12} \mathrm{O}_{6} \\
180\end{array}$ & + & $\begin{array}{c}\mathrm{C}_{6} \mathrm{H}_{12} \mathrm{O}_{6} \\
180\end{array}$ \\
\hline $\begin{array}{c}2 \mathrm{C}_{6} \mathrm{H}_{12} \mathrm{O}_{6} \\
360\end{array}$ & $\begin{array}{c}+12 \mathrm{O}_{2} \\
384\end{array}$ & $\rightarrow$ & $\begin{array}{l}12 \mathrm{CO}_{2} \\
528\end{array}$ & + & $\begin{array}{c}12 \mathrm{H}_{2} \mathrm{O} \\
216\end{array}$ \\
\hline
\end{tabular}

No segundo caso, conhecido como fermentação alcoólica, uma molécula de sacarose é oxidada na ausência de oxigênio, resultando em etanol e gás carbônico nas quantidades estequiométricas mostradas na equação 2 (LEHNINGER, 2002):

$$
\begin{array}{ccc}
\mathrm{C}_{12} \mathrm{H}_{22} \mathrm{O}_{11}+\mathrm{H}_{2} \mathrm{O} & \rightarrow \mathrm{C}_{6} \mathrm{H}_{12} \mathrm{O}_{6}+\mathrm{C}_{6} \mathrm{H}_{12} \mathrm{O}_{6} \\
342+18 & \rightarrow 180+180 \\
2 \mathrm{C}_{6} \mathrm{H}_{12} \mathrm{O}_{6} & \rightarrow & 4 \mathrm{C}_{2} \mathrm{H}_{5} \mathrm{OH}+4 \mathrm{CO}_{2} \\
360 & \rightarrow & 184+176
\end{array}
$$

Kocková-Kratochvílová (1990) afirmou que em algumas leveduras a respiração e a fermentação ocorrem na mesma extensão, enquanto que em outras um desses processos predomina. Também dividiu as leveduras em três grupos em função de sua capacidade respiratória: a) prevalência da respiração - leveduras forrageiras (a respiração é responsável por $100 \%$ do catabolismo); b) respiração e fermentação são semelhantes - leveduras patogênicas, de panificação e cervejeira de alta fermentação (a respiração corresponde a 40-50 \% do catabolismo); c) prevalência da fermentação - leveduras de destilaria, vinho e cervejeira de baixa fermentação (a respiração responde por $10-15 \%$ do catabolismo).

Briggs et al. (2004) relataram que leveduras facultativas podem ser classificadas como: tipo respiratório (mais de $70 \%$ do açúcar metabolizado por respiração) e tipo fermentativo (menos de $10 \%$ do açúcar é respirado). Esses autores enumeraram vários tipos de catabolismo de açúcar em leveduras: a) efeito Crabtree short-term - atenuação da respiração pela presença de glicose; b) efeito Crabtree long-term - repressão e inativação da respiração pela presença de glicose; c) efeito Pasteur - redução da glicólise sob condições aeróbias; d) efeito Kluyver - utilização aeróbia obrigatória de dissacarídeos; e) efeito Custers - estimulação aeróbia da fermentação da glicose.

Para Ingledew (2009), a concentração de açúcar no mosto das destilarias americanas de etanol é alta (mais de $0,1 \% \mathrm{~m} / \mathrm{v}$ ), fazendo com que as leveduras não sejam capazes de crescer sob condições aeróbias, mesmo na presença de aproximadamente $4 \mathrm{mg} / \mathrm{L}$ de oxigênio proveniente do ar acima da superfície do mosto. Assim, a glicose só pode ser transformada por metabolismo anaeróbio. Segundo esse autor, mais de $90 \%$ da glicose do mosto é metabolizada anaerobiamente mediante fermentação alcoólica, sendo o restante do açúcar usado no crescimento celular.

Em abordagem diferente, Munroe (1994) e Russell (2006) afirmaram que o oxigênio do mosto é usado pela levedura para a síntese de ácidos graxos insaturados e esterois, essenciais na síntese da membrana celular. Tais autores não mencionaram os efeitos Pasteur e Crabtree.

De acordo com Kocková-Kratochvílová (1990), os processos de respiração e fermentação 
podem ser mensurados manometricamente com base no consumo de oxigênio e na produção de gás carbônico. A relação entre fermentação e respiração é expressa pelo quociente respiratório $\left(Q_{R}\right)$, cujo valor varia conforme o tipo de levedura. Para dada cultura, os valores do quociente respiratório são mais elevados sob condições anaeróbias do que sob aerobiose. Os valores de $Q_{R}$ de 2 a 3 são mais frequentemente relatados para levedura de panificação; de 6 a 10 para levedura de vinho e de 10 a 30 para levedura de cervejeira de baixa fermentação.

Define-se o quociente respiratório pela equação 3 (FERNANDES, 2008):

$$
\mathrm{Q}_{\mathrm{R}}=\frac{\mathrm{CO}_{2} \text { produzido }}{\mathrm{O}_{2} \text { consumido }}
$$

Em trabalhos sobre a quantificação do metabolismo aeróbio e anaeróbio de leveduras alcoólicas foi utilizado o conceito de quociente respiratório $\left(Q_{R}\right)$. A presente pesquisa se propôs a quantificar o metabolismo aeróbio (respiração) e anaeróbio (fermentação alcoólica) de levedura alcoólica por meio de método estequiométrico, usando-se a determinação das massas de gás carbônico e etanol produzidos durante o processo fermentativo de mostos de melaço e caldo de cana.

\section{MATERIAL E MÉTODOS}

Os testes foram conduzidos com 3 tratamentos e 3 repetições, perfazendo 9 parcelas experimentais. Os tratamentos constituíram-se em diferentes concentrações de sólidos solúveis do mosto (10, 20 e $30^{\circ}$ Brix), sendo os mostos preparados a partir de melaço e de caldo de cana.

No preparo dos mostos, o melaço foi diluído com água deionizada (conforme a concentração final desejada), enquanto que para os mostos de caldo de cana empregou-se água deionizada ou açúcar cristal para atingir os teores de sólidos solúveis desejados. Para tanto, utilizou-se o balanço de massa de sólidos solúveis, conforme a equação 4 (BRUNELLI e VENTURINI FILHO, 2012):

$$
B_{1} \cdot M_{1}+B_{2} \cdot M_{2}=B_{3} \cdot M_{3}
$$

$\mathrm{B}_{1}=$ teor de sólidos solúveis do melaço ou do caldo de cana ( $\left.{ }^{\circ} \mathrm{Brix}\right)$;

$\mathrm{M}_{1}=$ massa do melaço ou do caldo de cana $(\mathrm{g})$;

$\mathrm{B}_{2}=$ teor de sólidos solúveis da água ou do açúcar cristal ('Brix);

$\mathrm{M}_{2}=$ massa da água ou do açúcar cristal $(\mathrm{g})$;

$\mathrm{B}_{3}=$ teor de sólidos solúveis do mosto ( $\left.{ }^{\circ} \mathrm{Brix}\right)$;

$\mathrm{M}_{3}=$ massa de mosto $(\mathrm{g})$.

Preparou-se 1,0 litro de mosto nas concentrações especificadas e inoculou-se fermento seco de panificação na proporção de $100 \mathrm{~g} / \mathrm{L}$. Usou-se béquer de 4 litros como fermentador e bastão plástico de agitação (parte constituinte do sistema de fermentação) para quebrar a espuma formada nas primeiras horas de fermentação (todos os tratamentos foram agitados exatamente da mesma forma). Na repetição 2, substituiu-se o bastão plástico por termômetro para mensurar a temperatura do mosto em fermentação. A fermentação transcorreu em temperatura ambiente, sendo efetuadas leituras horárias da massa de mosto em fermentação. Após a $10^{a}$ leitura interrompeu-se o experimento e determinou-se o teor alcoólico dos mostos fermentados (vinhos).

No mosto fermentado (vinho) analisou-se o teor alcoólico pelo método da destilação (destilador marca Buchi, modelo K355), procedeu-se a leitura da densidade do destilado (densímetro digital marca Mettler, modelo DA310) e converteu-se a densidade em teor alcoólico (\% v/v) por meio da tabela Porcentagem de álcool em volume a $20^{\circ} \mathrm{C}(\% \mathrm{~V} / \mathrm{V})$ correspondente à densidade relativa (BRASIL, 2005).

As massas de mosto em fermentação foram determinadas em balança de precisão (marca Gehaka, modelo BG2000). As massas de gás carbônico produzido no processo foram determinadas 
pela diferença de duas leituras consecutivas das massas de mosto em fermentação.

A quantificação do metabolismo aeróbio e anaeróbio da levedura durante o processo fermentativo foi estimada estequiometricamente (ATKINS e JONES, 2001) por meio da quantificação relativa da sacarose respirada e fermentada, conforme o seguinte protocolo.

Cálculo da sacarose fermentada (metabolismo anaeróbio):

a) Determinou-se o teor alcoólico do mosto fermentado;

b) Calculou-se a massa de etanol;

c) A partir da massa de etanol, calculou-se estequiometricamente (Equação 2) a massa de sacarose fermentada.

Cálculo da sacarose respirada (metabolismo aeróbio):

a) Mensurou-se a massa total de $\mathrm{CO}_{2}$ produzida no processo, proveniente tanto do metabolismo aeróbio como do anaeróbio;

b) Converteu-se estequiometricamente a massa de etanol formado na fermentação alcoólica em massa de gás carbônico (Equação 2), produzido pelo metabolismo anaeróbio;

c) Calculou-se a massa de $\mathrm{CO}_{2}$ proveniente da respiração mediante subtração da massa total de gás carbônico gerado no processo pela massa de gás carbônico produzido pelo metabolismo anaeróbio;

d) Converteu-se estequiometricamente a massa de $\mathrm{CO}_{2}$ proveniente do metabolismo respiratório em massa de sacarose respirada (Equação 1);

e) Obteve-se a massa total de sacarose catabolizada pela soma da massa de sacarose fermentada e da sacarose respirada.

Efetuou-se a quantificação relativa de sacarose fermentada e respirada por meio das equações 5 e 6 :

$$
\begin{aligned}
& \text { Sacarose fermentada (\%) }=\frac{M S F}{M S F+M S R} \\
& \text { Sacarose respirada }(\%)=\frac{M S R}{M S R+M S F}
\end{aligned}
$$

Em que:

MSF = massa de sacarose fermentada;

$\mathrm{MSR}=$ massa de sacarose respirada.

Como os tratamentos (teor de sólidos solúveis dos mostos) são quantitativos, a avaliação estatística foi realizada por meio de análise de variância da regressão linear, que relacionou os valores do Brix inicial dos mostos com as taxas de fermentação e respiração da levedura (VIEIRA, 2006). Os cálculos estatísticos foram efetuados com auxílio do programa Microsoft Office Excel 2007® (MICROSOFT Co., 2007).

\section{RESULTADOS E DISCUSSÃO}

As Figuras 1a e 1b mostram a taxa horária de produção de gás carbônico e a evolução da temperatura durante o processo fermentativo de mosto de melaço. Observou-se que para mosto com $10{ }^{\circ}$ Brix, a produção máxima de $\mathrm{CO}_{2}(22,79 \mathrm{~g} / \mathrm{L}$.h $)$ ocorreu na primeira hora de fermentação, enquanto que para os mostos com 20 e $30^{\circ}$ Brix, a máxima produção ocorreu na segunda hora de fermentação (40,43 e 29,24 g/L.h, respectivamente). Notou-se também que no início do processo, a fermentação do mosto com $30^{\circ}$ Brix foi mais lenta em função do efeito osmótico, causado pelo excesso de açúcar. $\mathrm{O}$ mosto com $20^{\circ} \mathrm{Brix}$ resultou em fermentação mais produtiva.

Iniciou-se a fermentação com temperatura em torno de $28^{\circ} \mathrm{C}$ para todos os tratamentos. $\mathrm{O}$ pico de temperatura foi atingido na segunda hora para o mosto com $10^{\circ} \mathrm{Brix}\left(32,0^{\circ} \mathrm{C}\right)$, na terceira 
hora para o mosto com $20^{\circ}$ Brix $\left(32,0^{\circ} \mathrm{C}\right)$ e na quarta hora para o mosto com $30{ }^{\circ}$ Brix $\left(34,5^{\circ} \mathrm{C}\right)$. Observou-se defasagem de uma hora entre a máxima atividade metabólica da levedura e o pico de temperatura do mosto em fermentação.
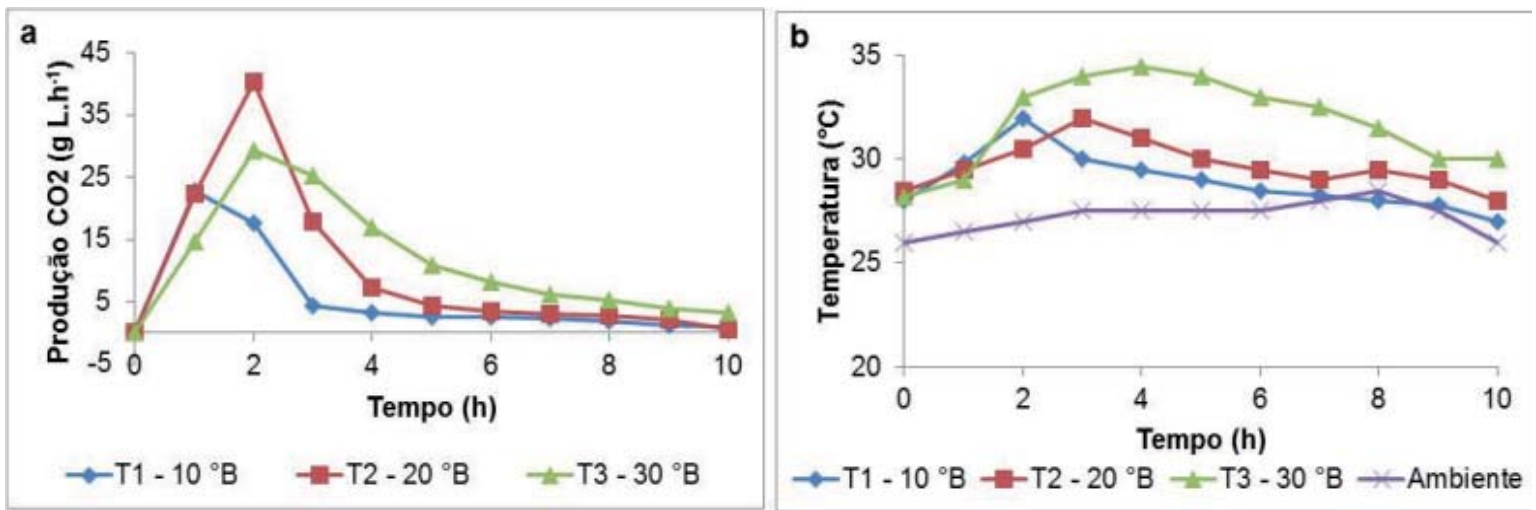

\section{FIGURA 1 - TAXA HORÁRIA DE PRODUÇÃO DE GÁS CARBÔNICO (A) E EVOLUÇÃO DA TEMPERATURA DURANTE O PROCESSO FERMENTATIVO (B) DO MOSTO DE MELAÇO}

A forma das curvas de produção de $\mathrm{CO}_{2}$ para os mostos de caldo de cana mostrou-se semelhante a dos mostos de melaço. No entanto, a fermentação para o mosto de caldo de cana foi mais lenta. Isso não deve ser creditado ao tipo de matéria-prima empregada no preparo do mosto, mas ao fato do teste com mosto de caldo de cana ser iniciado em temperatura inferior $\left(22,0^{\circ} \mathrm{C}\right)$.

A maior produção de gás carbônico, tanto para os mostos de melaço como para os de caldo de cana, ocorreu nas primeiras horas de fermentação (Figuras 1a e 2a). Como será visto mais adiante, a levedura metabolizou aerobicamente uma parcela do açúcar dos mostos (melaço e caldo de cana) e necessariamente isso ocorreu no início da fermentação quando havia oxigênio dissolvido nesses meios.
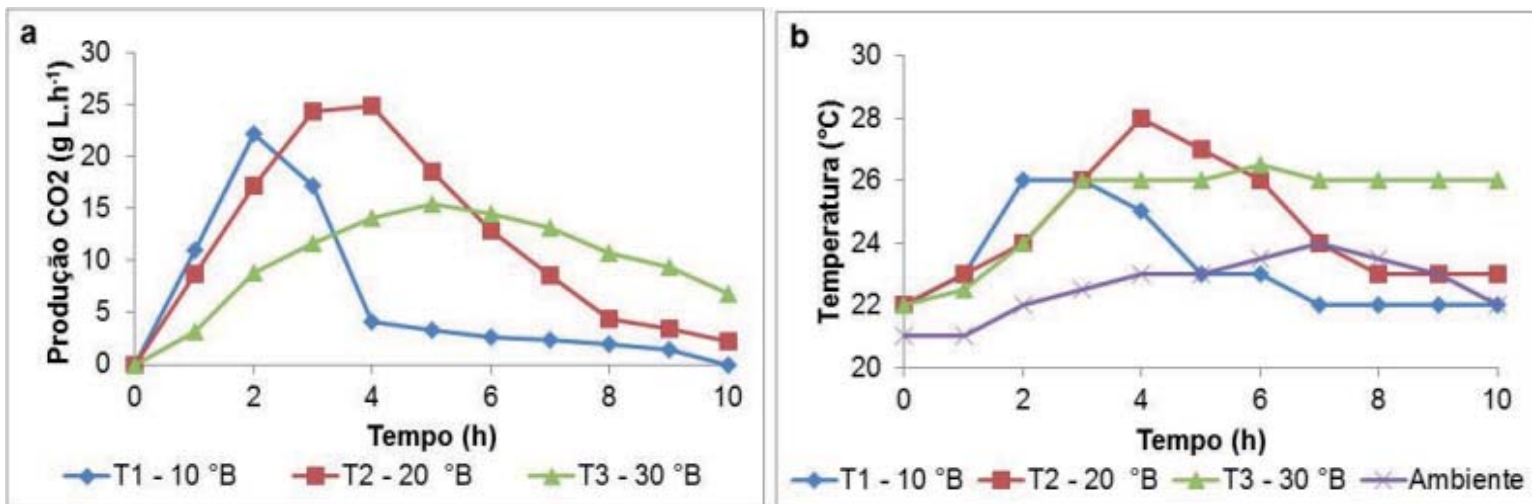

\section{FIGURA 2 - TAXA HORÁRIA DE PRODUÇÃO DE GÁS CARBÔNICO (A) E EVOLUÇÃO DA TEMPERATURA DURANTE O PROCESSO FERMENTATIVO (B) DO MOSTO DE CALDO DE CANA}

Os resultados da quantificação relativa dos metabolismos aeróbio e anaeróbio da levedura durante o processo fermentativo em mosto de melaço são apresentados nas Tabelas 1 e 2 , enquanto que as Tabelas 3 e 4 mostram os resultados da fermentação do mosto de caldo de cana. Os dados das Tabelas 1 e 3 são apresentados na forma sequencial dos cálculos efetuados.

Nos cálculos, utilizou-se a sacarose como açúcar de referência em ambos os mostos, 
uma vez que esse açúcar prevalece tanto no caldo de cana como no de melaço. De acordo com a Copersucar (1987), o caldo de cana tem concentrações de sacarose, glicose e frutose de $14 \%$, $0,2 \%$ e $0,4 \%$, respectivamente, enquanto que o melaço apresenta por volta de $39 \%$ de sacarose e $9,5 \%$ de açúcares redutores (glicose e frutose).

\section{TABELA 1 - QUANTIFICAÇÃO DA SACAROSE FERMENTADA E RESPIRADA DURANTE O PROCESSO DE FERMENTAÇÃO DO MOSTO DE MELAÇO}

\begin{tabular}{|c|c|c|c|}
\hline & \multicolumn{3}{|c|}{ Mosto ( ${ }^{\circ}$ Brix) } \\
\hline & 10 & 20 & 30 \\
\hline Teor alcoólico vinho (\% v/v) & 4,4 & 9,0 & 12,8 \\
\hline Volume etanol vinho $(\mathrm{mL})$ & 44,42 & 89,53 & 127,55 \\
\hline Massa etanol vinho $(\mathrm{g})$ & 35,09 & 70,73 & 100,76 \\
\hline Massa $\mathrm{CO}_{2}$ metabolismo anaeróbio $(\mathrm{g})$ & 33,53 & 67,58 & 96,28 \\
\hline Massa sacarose fermentada $(\mathrm{g})$ & 66,67 & 134,39 & 191,45 \\
\hline Massa $\mathrm{CO}_{2}$ fermentação + respiração (g) & 59,27 & 104,42 & 123,59 \\
\hline Massa $\mathrm{CO}_{2}$ respiração $(\mathrm{g})$ & 25,74 & 36,84 & 27,31 \\
\hline Massa sacarose respirada (g) & 16,65 & 23,82 & 17,62 \\
\hline Massa sacarose fermentada + respirada $(\mathrm{g})$ & 83,32 & 158,21 & 209,07 \\
\hline Sacarose fermentada (\%) & 80,02 & 84,95 & 91,58 \\
\hline Sacarose respirada (\%) & 19,98 & 15,05 & 8,42 \\
\hline
\end{tabular}

A análise de regressão (Tabela 2) mostrou que a taxa de fermentação (porcentagem de sacarose fermentada) relacionou-se com o Brix inicial do mosto de melaço por meio de equação linear $(y=0,5871 x+73,467)$ de coeficiente angular positivo $(0,5871)$ e com elevado coeficiente de determinação $\left(R^{2}=0,9926\right)$. O teste $F$ para a regressão mostrou-se significativo, sendo o valor de $F$ calculado $(210,58)$ maior que o valor de $F$ crítico $\left(p=1,76.10^{-6}\right)$, o que demonstra com clareza a relação de proporcionalidade entre o Brix inicial do mosto e a taxa de fermentação da levedura. $A$ mesma análise pode ser efetuada para a taxa de respiração (porcentagem de sacarose respirada). Nesse caso, a taxa de respiração relacionou-se com o teor de sólidos solúveis do mosto de melaço por meio de equação linear $(y=-0,5871 x+26,533)$ de coeficiente linear negativo $(-0,5871)$ e com elevado coeficiente de determinação $\left(R^{2}=0,9926\right)$. O teste $F$ para a regressão mostrou-se significativo, sendo o valor de $F$ calculado $(210,58)$ maior que o valor de $F$ crítico $\left(p=1,76.10^{-6}\right)$, demonstrando a relação inversa entre o teor de sólidos solúveis do mosto e a taxa de respiração do fermento.

\section{TABELA 2 - ANÁLISE DE VARIÂNCIA DA REGRESSÃO LINEAR (FERMENTAÇÃO DO MOSTO DE MELAÇO)}

\begin{tabular}{cccccc}
\hline & GL & SQ & QM & F & $p$ \\
\hline Regressão & 1 & 200,34 & 200,34 & 210,86 & 1,76 E-06 \\
\hline Resíduo & 7 & 6,65 & 0,95 & & \\
\hline Total & 8 & 206,99 & & & \\
\hline
\end{tabular}

$\mathrm{GL}=$ graus de liberdade; $\mathrm{SQ}=$ Quadrado total; $\mathrm{QM}=$ Quadrados médio; $\mathrm{F}=$ Valor $\mathrm{F}$ calculado; $\mathrm{p}=\mathrm{p}$-valor. 
Os coeficientes angulares das equações são iguais em módulo $(0,5871)$, bem como os coeficientes de determinação, os valores de $\mathrm{F}$ calculado e $\mathrm{F}$ crítico. Isso ocorreu em virtude da taxa de fermentação apresentar relação inversa com a taxa de respiração (taxa de fermentação $=100-$ taxa de respiração).

\section{TABELA 3 - QUANTIFICAÇÃO DA SACAROSE FERMENTADA E RESPIRADA DURANTE O PROCESSO DE FERMENTAÇÃO DO MOSTO DE CALDO DE CANA}

\begin{tabular}{|c|c|c|c|}
\hline & \multicolumn{3}{|c|}{ Mosto ( ${ }^{\circ}$ Brix $)$} \\
\hline & 10 & 20 & 30 \\
\hline Teor alcoólico vinho (\% v/v) & 5,47 & 10,95 & 10,47 \\
\hline Volume etanol vinho (mL) & 54,73 & 109,50 & 104,73 \\
\hline Massa etanol vinho $(\mathrm{g})$ & 43,24 & 86,51 & 82,74 \\
\hline Massa $\mathrm{CO}_{2}$ fermentação $(\mathrm{g})$ & 41,31 & 82,65 & 79,06 \\
\hline Massa sacarose fermentada $(\mathrm{g})$ & 80,32 & 160,69 & 153,69 \\
\hline Massa $\mathrm{CO}_{2}$ fermentação + respiração (g) & 66,40 & 125,18 & 107,92 \\
\hline Massa $\mathrm{CO}_{2}$ respiração $(\mathrm{g})$ & 25,09 & 42,52 & 28,86 \\
\hline Massa sacarose respirada $(\mathrm{g})$ & 16,26 & 27,56 & 18,70 \\
\hline Massa sacarose fermentada + respirada $(\mathrm{g})$ & 96,58 & 188,25 & 172,40 \\
\hline Sacarose fermentada (\%) & 83,17 & 85,36 & 89,16 \\
\hline Sacarose respirada $(\%)$ & 16,83 & 14,64 & 10,84 \\
\hline
\end{tabular}

A análise de regressão (Tabela 4) dos dados referentes à fermentação do mosto de caldo de cana apresentou resultados similares à fermentação do mosto de melaço. Essa análise revelou que a porcentagem de sacarose fermentada relacionou-se com o teor de sólidos solúveis dos mostos de caldo de cana por meio de equação linear $Y=0,2997 X+79,905\left(R^{2}=0,9047\right)$, enquanto para a respiração da sacarose obteve-se a equação $Y=-0,2997 X+20,095\left(R^{2}=0,9047\right)$.

\section{TABELA 4 - ANÁLISE DE VARIÂNCIA DA REGRESSÃO LINEAR (FERMENTAÇÃO DO MOSTO DE CALDO DE CANA)}

\begin{tabular}{cccccc}
\hline & GL & SQ & QM & F & p \\
\hline Regressão & 1 & 52,69 & 52,69 & 63,54 & $9,33 E-05$ \\
\hline Resíduo & 7 & 5,81 & 0,83 & & \\
\hline Total & 8 & 58,50 & &
\end{tabular}

$\mathrm{GL}=$ graus de liberdade; $\mathrm{SQ}=$ Quadrado total; $\mathrm{QM}=$ Quadrados médio; $\mathrm{F}=$ Valor F calculado; $\mathrm{p}=\mathrm{p}$-valor .

Observam-se nas Tabelas 1 e 3 que a porcentagem de sacarose fermentada aumentou com a elevação do teor de sólidos solúveis no mosto, ocorrendo o inverso com o percentual 
de açúcar respirado. Esses resultados evidenciam o efeito Crabtree durante o processo. De acordo com Briggs et al. (2004), o efeito Crabtree observado na presente pesquisa enquadra-se no tipo short-term, isto é, a presença de açúcar no meio atenua, mas não inibe a respiração.

O efeito Crabtree observado neste trabalho não se comportou qualitativamente (ausência de respiração na presença de elevadas concentrações de açúcar no mosto), conforme previsto por Ingledew (2009), mas quantitativamente devido à relação de proporcionalidade entre a concentração de açúcar do mosto e o metabolismo anaeróbio (quanto maior a concentração de açúcar do mosto, maior a quantidade relativa de açúcar fermentado).

Na prática industrial, o efeito Crabtree tornou-se bem conhecido nas destilarias brasileiras de etanol que fazem uso de mostos diluídos para favorecer a multiplicação (respiração) de fermento no início da safra. Também ocorre quando a destilaria encontra dificuldades em refrigerar o mosto em fermentação. A fim de diminuir a geração de calor, a concentração inicial de sólidos solúveis do mosto é reduzida promovendo aumento no crescimento do fermento.

O percentual de açúcar respirado pela levedura nos mostos elaborados com melaço variou de 8,42 (30 ${ }^{\circ} \mathrm{Brix}$ ) a 19,98 (10 ${ }^{\circ} \mathrm{Brix}$ ) e nos mostos de caldo de cana de 10,84 (30 ${ }^{\circ} \mathrm{Brix}$ ) a 16,83 (30 ${ }^{\circ}$ Brix). Esses resultados confrontam os de Kocková-Kratochvílová (1990), que afirmou que a levedura de panificação apresenta $40-50 \%$ de metabolismo aeróbio. Essa divergência de resultados pode ter origem nas cepas de leveduras empregadas, nos diferentes métodos de quantificação utilizados, além das condições ambientais distintas em que os testes foram conduzidos.

Acredita-se que o gás carbônico produzido no processo de fermentação provém fundamentalmente do catabolismo aeróbio e anaeróbio da levedura. No entanto, o açúcar assimilado pela levedura também é usado em outras reações metabólicas, como as do anabolismo (síntese de compostos celulares), das quais resulta o crescimento da população do micro-organismo. Ingledew (2009) citou que $10 \%$ do açúcar presente no mosto são usados para o crescimento das leveduras alcoólicas.

Deve-se reconhecer que o método estequiométrico proposto, para quantificar o catabolismo aeróbio e anaeróbio da levedura alcoólica durante o processo fermentativo, pode apresentar pequeno erro inerente à metodologia em função da incerteza de que todo $\mathrm{CO}_{2}$ produzido tenha origem exclusivamente na fermentação alcoólica e respiração da levedura. Assim, o método apenas estima o metabolismo aeróbio e anaeróbio da levedura alcoólica. Entretanto, o excelente ajuste das equações de regressão $\left(R^{2}\right)$ e a significância estatística elevada evidenciam que o método proposto é confiável, confirmando experimentalmente os resultados observados por outros autores utilizando metodologia distinta (efeito Crabtree).

Devido a sua simplicidade, o método proposto pode ser aplicado com finalidade didática (aulas práticas de cursos envolvidos com a fermentação alcoólica), de pesquisa e até mesmo em empresas do setor sucroalcooleiro e de bebidas alcoólicas interessadas em conhecer melhor as características metabólicas de seus fermentos.

\section{CONCLUSÃO}

As condições em que o trabalho experimental foi realizado permitem concluir que:

- Durante o seu cultivo, as leveduras de panificação catabolizam o açúcar do mosto de melaço e de caldo de cana, tanto pelo metabolismo aeróbio como pelo anaeróbio, com prevalência do segundo;

- Quanto maior for a concentração de açúcares nos mostos de melaço e de caldo de cana, maior será a atividade metabólica anaeróbia e menor a do metabolismo aeróbio; 
- O efeito Crabtree observado apresenta comportamento quantitativo (atividade metabólica anaeróbia proporcional à concentração inicial de sólidos solúveis do mosto) e não qualitativo (ausência de metabolismo aeróbio em mosto com elevada concentração de açúcares).

Pela sua simplicidade, o método proposto pode ser usado pelas destilarias brasileiras de etanol, cervejarias e indústrias correlatas para melhor caracterizar seus fermentos, bem como ser aproveitado nas atividades de ensino de cursos de graduação e pós-graduação que têm na fermentação alcoólica seu objeto de estudo.

\section{ABSTRACT \\ SIMPLE METHOD FOR QUANTIFICATION OF AEROBIC AND ANAEROBIC METABOLISM OF ALCOHOLIC YEAST}

The objective of this work was to quantify aerobic (respiration) and anaerobic (alcoholic fermentation) metabolisms of yeasts during the fermentation of two musts made from sugarcane or molasses by using one simple stoichiometric method. The experiment was conducted on three treatments (soluble solids content for the musts) and three repetitions adding up to nine parcels for each type of must (molasses and sugarcane juice). Molasses were diluted into water producing musts with 10, 20 e $30^{\circ}$ Brix, while for sugarcane either water or granulated sugar was added to the juice for the same purpose. Musts were inoculated using dry bakery yeast $(100 \mathrm{~g} / \mathrm{L})$ and let to ferment under room temperature. $\mathrm{CO}_{2}$ and ethanol masses were determined throughout all the fermentation to quantify metabolized sugars (fermented and respired), using stoichiometric calculations. Results showed that during the process, yeasts catabolized sugar by both aerobic and anaerobic pathways, whereas the anaerobic prevailed. Fermentation rate, for the molasses must, ranged from $80.02 \%\left(10^{\circ} \mathrm{Brix}\right)$ to $91.58 \%\left(30{ }^{\circ}\right.$ Brix) while for the sugarcane, from $83.17 \%\left(10^{\circ} \mathrm{Brix}\right)$ to $89.16 \%$ (30 $\left.{ }^{\circ} \mathrm{Brix}\right)$, which indicates that the Crabtree effect was present.

KEY-WORDS: FERMENTATION; YEAST; PASTEUR EFFECT; CRABTREE EFFECT.

\section{REFERÊNCIAS}

1 ATKINS, P.; JONES, L. Princípios de química: questionando a vida moderna e o meio ambiente. Porto Alegre: Bookman, 2001. $911 \mathrm{p}$.

2 BRASIL. Ministério da Saúde. Agência Nacional de Vigilância Sanitária. Métodos físico-químicos para análise de alimentos. 4 ed. Brasília, 2005. 1018 p.

3 COPERSUCAR. Fermentação. Piracicaba: Centro de Tecnologia Copersucar, 1987. 434 p.

4 BRIGgS, D. E.; BOULTON, C. A.; BROOKES, P. A.; STEVENS, A. Metabolism of wort by yeast. In: science and practice. Cambridge: Woodhead Publishing, 2004. p.401-468. Brewing:

5 BRUNELLI, L. T.; VENTURINI FILHO, W. G. Bebida mista de soja e uva. Alimentos e Nutrição, Araraquara, v.23, n.3, p.467-473, 2012.

6 FERNANDES, A. P. F. V. Leveduras isoladas de produtos frutícolas: capacidade fermentativa e estudos sobre a $\mathrm{H}^{+}$-ATPase da membrana plasmática. 2008. 201 f. Tese (Doutorado em Biologia, área de concentração Microbiologia) Universidade Nova de Lisboa, Faculdade de Ciências e Tecnologia, Lisboa, 2008.

7 INGLEDEW, W. M. Yeast: physiology, nutrition and ethanol production. In: INGLEDEW, W. M.; KELSALL, D. R.; AUSTIN, G. D.; KLUHSPIES, C. The alcohol textbook. $5^{\text {th }}$ ed. Nottingham: Nottingham University Press, 2009. p.101-113.

8 KOCKOVÁ-KRATOCHVÍLOVÁ, A. Yeast metabolism. In: Yeast and yeast-like organisms. Weinheim: $\mathrm{VCH}$, 1990. p. 304-390.

9 LEHNINGER, A. Princípios da bioquímica. 3. ed. São Paulo: Sarvier, 2002.

10 MICROSOFT CORPORATION. Microsoft office excel. Redmond, WA, 2007.

11 MUNROE, J. H. Fermentation. In: HARDWICK, W. A. Handbook of brewing. New York: Marcel Dekker, 1994. p.323353.

12 RUSSELL, I. Yeast. In: PRIEST, F. G.; STEWART G. G. Handbook of brewing. Boca Raton: Taylor \& Francis, 2006. p. 
281-332.

13 VIEIRA, S. Análise de variância (ANOVA). São Paulo: Atlas, 2006. 204 p.

AGRADECIMENTOS

FAPESP - Processo 2011/05669-0 